Research article

\title{
The estimated economic burden of genital herpes in the United States. An analysis using two costing approaches Thomas D Szucs ${ }^{1}$, Karin Berger ${ }^{2}$, David N Fisman ${ }^{3}$ and Stephan Harbarth*4
} \begin{abstract}
Hospital, Boston, USA
E-mail: Thomas D Szucs - Thomas.Szucs@hirslanden.ch; Karin Berger - merg.munich@t-online.de; David N Fisman - dfisman@caregroup.harvard.edu; Stephan Harbarth* - harbarth@post.harvard.edu ${ }^{*}$ Corresponding author
\end{abstract}

Address: ${ }^{1}$ Department of Medical Economics, University Hospital, Zurich, Switzerland, ${ }^{2}$ Medical Economics Research Group, Munich, Germany, ${ }^{3}$ Harvard Center for Risk Analysis, Harvard School of Public Health, Boston, USA and ${ }^{4}$ Division of Infectious Diseases, Children's

Published: 28 June 2001

Received: 26 April 2001

BMC Infectious Diseases 200I, I:5

Accepted: 28 June 2001

This article is available from: http://www.biomedcentral.com//47/-2334/I/5

(C) 200I Szucs et al, licensee BioMed Central Ltd.

\begin{abstract}
Background: Only limited data exist on the costs of genital herpes (GH) in the USA. We estimated the economic burden of GH in the USA using two different costing approaches.

Methods: The first approach was a cross-sectional survey of a sample of primary and secondary care physicians, analyzing health care resource utilization. The second approach was based on the analysis of a large administrative claims data set. Both approaches were used to generate the number of patients with symptomatic GH seeking medical treatment, the average medical expenditures and estimated national costs. Costs were valued from a societal and a third party payer's perspective in 1996 US dollars.

Results: In the cross-sectional study, based on an estimated 3.I million symptomatic episodes per year in the USA, the annual direct medical costs were estimated at a maximum of $\$ 984$ million. Of these costs, $49.7 \%$ were caused by drug expenditures, $47.7 \%$ by outpatient medical care and $2.6 \%$ by hospital costs. Indirect costs accounted for further $\$ 214$ million. The analysis of $1,565 \mathrm{GH}$ cases from the claims database yielded a minimum national estimate of $\$ 283$ million direct medical costs.

Conclusions: $\mathrm{GH}$ appears to be an important public health problem from the health economic point of view. The observed difference in direct medical costs may be explained with the influence of compliance to treatment and possible undersampling of subpopulations in the claims data set. The present study demonstrates the validity of using different approaches in estimating the economic burden of a specific disease to the health care system.
\end{abstract}

\section{Introduction}

Herpes simplex virus type 2 (HSV-2) is the most frequent causative organism of genital herpes $(\mathrm{GH})$ in the United States, while HSV type 1 is felt responsible for this recurrent infection in only 20 to 30 percent of cases [1]. GH is contagious both in the symptomatic and in the asymptomatic phase of the disease and causes painful genital ulcers. The management of the patient involves consultations, laboratory exams and drug treatment for the disease and its complications.

GH is one of the three most widespread sexually transmitted diseases in the USA [2]. Published data show that 45 million persons aged 12 years or older have HSV-2 an- 
tibodies [3], that up to $70 \%$ of patients attending sexual transmitted disease clinics have HSV-2 infection [4] and that the majority of patients with initially symptomatic GH will develop recurrent disease [5].

Cost of illness studies represent one of the applications of economic science to medicine. The aim of these studies is to assess the economic burden of a disease and to help decision-makers in targeting preventive efforts and allocating resources $[6,7]$. Despite its widespread and increasing transmission, there is still poor understanding of the economic impact of GH in the USA, which makes it difficult to evaluate societal costs and the cost-effectiveness of preventive efforts [8]. Therefore, the objective of the present study was to estimate the economic burden of GH in the USA, using two different costing approaches.

\section{Methods}

We conducted a population-based study on the costs of $\mathrm{GH}$. In order to give a better estimate of the disease burden, we retrieved economic information using two different approaches: In the first instance, direct interviews were conducted with a random sample of office-based physicians using a structured, comprehensive questionnaire. Secondly, information was retrieved from a large, longitudinal administrative database. For this purpose, data from Diversified Pharmaceutical Services (DPS), which is a pharmaceutical benefit management firm, were used. For both approaches, the time span was one year (1996). Costs were referred to on a yearly basis and computed in 1996 US dollars. Data were collected and analyzed separately.

\section{Approach using expert interviews}

A questionnaire was administered to 30 randomly selected primary and secondary care physicians practicing in the North-East of the USA. Physicians were asked about the annual number of patients with GH and the total number of episodes of symptomatic GH, seeking medical care and treatment. Data collection included the following variables: (1) demographic characteristics of the physicians' practices; (2) epidemiological figures: number of patients with $\mathrm{GH}$ per year; stratification of patients according to age, gender, severity of disease recurrence rates; duration of episodes; and (3) resource utilization: frequency of consultations and laboratory tests for GH patients; prescription of drugs in first/recurrent episodes and duration of treatment; frequency of hospitalizations due to GH complications; employment of patients; proportion of patients unable to work.

The perspective of this analysis was societal; thus, both direct and indirect medical costs were based on the costs born by society. The US population in 1996 (279 million) served as a reference. Regarding medical costs, the categories considered were the consultations and medical procedures performed, laboratory tests, pharmacological treatment and hospitalizations. Unit costs are illustrated in Table 1. For the purpose of this analysis, average monetary values from all respondents were considered [9]. Values used came directly from interviews with physicians involved in the study.

Indirect costs were calculated using the human capital approach $[7,10,11]$. These costs were based on an average U.S. hourly wage of $\$ 14$ [12], and were valued according to estimates of time consumed by hospitalization, time lost from work due to illness, and travel and waiting-room time resulting from physician visits. We estimated that individuals with a primary $\mathrm{GH}$ syndrome would miss two days of work if symptoms were not severe enough to require hospitalization, and one week of work if hospitalization was required. Patient travel and waiting room time was estimated to be two hours per physician visit. We did not use gender-specific wage rates, as females are underpaid relative to men [12], so that gender specific wage-rates would implicitly undervalue the costs of GH in females.

\section{Approach using claims database}

DPS is a pharmaceutical benefit service collecting and processing claims on consultations, laboratory tests, drug utilization and hospital treatment from Health Maintenance Organizations (HMOs) and Independent Practice Associations from different areas in the USA. For the present study, all patients diagnosed with GH who have been enrolled in the plans of four HMOs at any time during 1996 were included. The four health plans included in the analysis were located in the Southwest, East, Mid-West, and West of the USA. The approximately 0.5 million members enrolled in the health plans were generally employed or dependents of employed members. The majority of members reside in an urban rather than rural environment, representing an urban, working-class population. Less well represented in this population were the elderly (age over 65), the unemployed

Table I: Unit costs per episode used in the physicians' study

\begin{tabular}{ll}
\hline Variable & Unit co \\
\hline & \\
\hline Consultation & 73.00 \\
Clinical examination & 40.33 \\
Microbiologic evaluation & 38.39 \\
Complete blood count & 21.29 \\
Antibody testing & 76.50 \\
Urine analysis & 12.59 \\
Hospital day & 669.00 \\
Days off work & 144.00
\end{tabular}


and those entitled to state medical assistance (6\% of the plan members had Medicaid as their principal insurance).

Members were included if they had at least one claim for $\mathrm{GH}$ during the study period. We used the following International Classification of Disease-9 codes for data collection: 054.1 (GH); 054.10 (GH, unspecified); 054.11 (herpetic vulvovaginitis); 054.12 (herpetic ulceration of vulva); 054.13 (herpetic infection of penis) and 054.19 (other GH). For this analysis, the following definitions were used: "prevalent case" = members who had a GH diagnosis claim between the dates of $1 / 1 / 96$ and 12/31/ 96; "active case" = members who had a GH diagnosis claim between the dates of $1 / 1 / 96$ and 12/31/96, and had a drug claim for treating $\mathrm{GH}$ or were treated in the hospital for GH; "incident case" = members who had a GH diagnosis or drug claim in 1996 and no GH claim prior to 1996; "recurrent case" = members who were continuously enrolled in 1996, had a GH diagnosis or drug claim in 1996 and a recurrent claim 15 days or more after the first claim (incident cases excluded).

Direct medical costs were based on actual pharmacy, outpatient and hospital claims processed by DPS. Component costs were based on drug costs, outpatient costs, emergency room costs, inpatient costs, laboratory costs and home visit costs. Drugs used for treatment of GH were identified in members who also had a diagnosis claim for $\mathrm{GH}$ at some time during the study period. This allows for some reliance that the drug was used to treat GH and not some other form of herpetic infection. Mean, median, and standard deviation were calculated to describe the population statistics for costs. The perspective of this analysis was third party payer.

The annual costs attributable to GH-infection in the United States were estimated as the product of the number of incident and prevalent infections and the average present value of the costs attributable to a single $\mathrm{GH}$-infection. We also evaluated the impact of other GHassociated complications such as neonatal herpes and excess cesarean sections. A crude estimate of the burden of these complications was calculated, based on data extracted from the DPS database and published data $[1$, $13,14,15,16]$.

\section{Results}

\section{Approach using expert opinion}

In the sample of 30 interviewed physicians, 15 were general practitioners (GPs), while 15 were specialists ( 6 dermatologists, 4 gynecologists, 3 infectious disease specialists and 2 urologists). Based on our data, GPs see on average $34 \mathrm{GH}$ patients a year; among these patients $13(38 \%)$ were reported to be primary cases. Specialists see $56 \mathrm{GH}$ patients per year, including 12 (21\%) incident cases. Sixty-five percent of patients were in the 18-30 age group; $52 \%$ of patients were female. Almost half of the patients (49\%) suffered from mild GH, whereas moderate and severe cases represented $37 \%$ and $14 \%$ of patients, respectively. Forty-eight percent of patients experienced less than 2 relapses a year, $36 \% 2$ to 5 relapses and $16 \%$ more than 5 per year. A typical first GH episode was reported to last on average 10.8 days, slightly more than a typical recurrence (8.4 d). From the data available, the incidence of clinically manifest GH can be estimated to be 423,000 cases and the number of recurrent cases at about 698,000 patients in 1996. These estimates correspond to an occurrence rate of 3,139,000 symptomatic episodes.

Table 2 illustrates the total cost of illness obtained translating epidemiological figures and utilization rates in monetary terms using the unit cost values previously described. The estimated total burden of medical care for GH in the US in 1996 represents $\$ 984$ million, based on an estimated occurrence rate of 3.1 Mio symptomatic episodes. Of these costs, $49.7 \%$ were caused by drug expenditures, $47.7 \%$ by medical care and $2.6 \%$ by hospital costs. Further \$214 million represent the indirect costs to society. Thus, up to $\$ 1.2$ billion can be considered as being the total burden of GH to the US as computed with this methodology. Table 3 gives a breakdown of the costs at the single patient level.

\section{Approach using claims database}

Among 1,565 patients with GH, 65\% of patients were in the $21-40$ age group; $74 \%$ were female. Table 4 summarizes the main epidemiologic findings from this analysis. We extrapolated the collected data to the US population in 1996 (279 million) and calculated an annual incidence of 131,130 symptomatic $\mathrm{GH}$ cases in 1996, corresponding to a crude incidence of 0.47 per 1,000 . The total number of persons with prevalent, active or recurrent symptomatic GH was estimated to be 806,310 . This produces a crude prevalence of 2.89 cases per 1,000 in 1996.

Table 5 shows the costs of GH among DPS database enrollees. Costs were highest among recurrent cases and lowest among prevalent, symptomatic cases not requiring treatment. Using this approach, the amount of medical resources absorbed by the care of GH patients in the US can thus be estimated at \$244 million for 1996.

Based on previously published data $[1,13,14,15,16]$, we also estimated the direct costs of GH-associated complications. Neonatal herpes accounted for 400 annual cases with mean medical costs of $\$ 60,000$, whereas 6,000 excess cesarean sections contributed, on average, $\$ 2,500$ per case. Thus, the average cost of these $\mathrm{GH}$-associated 
complications can be estimated at \$39 million for 1996, bringing the total of the direct medical cost estimates to a sum of $\$ 283$ million.

\section{Discussion}

This report presents crude estimates rather than precise measures of the economic costs of GH in the USA. Using two different approaches, we estimated the total direct medical costs of GH to range from a minimum of $\$ 283$ million to a maximum of $\$ 984$ million in 1996. Indirect costs to society amounted to $\$ 214$ million due to production losses. Office-based medical care and drug treatment were the major sources of direct costs. Moreover, this study showed that the average GH patient seeking treatment is likely to be younger than 40 , to develop about 2 recurrent episodes, to undergo several laboratory exams, and to be treated with antiviral drugs. Relapses tended to be shorter than first episodes though having a higher cost per episode.

Our results are likely to be conservative estimates due to the great number of asymptomatic and shedding patients not seeking medical care and transmitting the disease to other individuals. As recently shown, the great majority of people with serologic evidence of HSV-2 infection have no history of recognized GH [3]. However, many seropositive persons shed HSV-2 that is detectable by culture from the genital tract, and many have symptoms that are directly referable to HSV-2 detectable by culture [17].

Table 2: Estimated costs of genital herpes in the USA (physicians' study)

\begin{tabular}{|c|c|c|c|}
\hline & $\begin{array}{c}\text { Costs (in } \\
\text { thousands US\$) }\end{array}$ & $\%$ of direct costs & $\%$ of total costs \\
\hline \multicolumn{4}{|c|}{ Consultations/lab testing $(n)$} \\
\hline First episodes $(422,950)$ & 74,016 & & \\
\hline I to $2(104,150)$ & 19,684 & & \\
\hline 3 to $6(468,650)$ & 265,725 & & \\
\hline 7 or more $(124,970)$ & 110,224 & & \\
\hline Subtotal & 469,649 & $47.7 \%$ & \\
\hline \multicolumn{4}{|l|}{ Drugs (n) } \\
\hline First episodes $(422,950)$ & 27,069 & & \\
\hline I to $2(104,150)$ & 20,465 & & \\
\hline 3 to $6(468,650)$ & 303,685 & & \\
\hline 7 or more $(124,970)$ & 137,342 & & \\
\hline Subtotal & 488,561 & $49.7 \%$ & \\
\hline \multicolumn{4}{|l|}{ Hospital (n) } \\
\hline First episodes $(422,950)$ & 424 & & \\
\hline I to $2(104,150)$ & 1,250 & & \\
\hline 3 to $6(468,650)$ & $|6,87|$ & & \\
\hline 7 or more $(124,970)$ & 6,998 & & \\
\hline Subtotal & 25,543 & $2.6 \%$ & \\
\hline \multicolumn{4}{|l|}{ Total direct costs $(n)$} \\
\hline First episodes $(422,950)$ & 101,508 & & \\
\hline I to $2(104,150)$ & 41,400 & & \\
\hline 3 to $6(468,650)$ & 586,281 & & \\
\hline 7 or more $(124,970)$ & 254,654 & & \\
\hline Total & 983,843 & $100 \%$ & $82.1 \%$ \\
\hline \multicolumn{4}{|l|}{ Total indirect costs $(n)$} \\
\hline First episodes $(422,950)$ & 25,377 & & \\
\hline I to $2(104,150)$ & 9,373 & & \\
\hline 3 to $6(468,650)$ & 126,536 & & \\
\hline 7 or more $(124,970)$ & 52,487 & & \\
\hline Total & 213,773 & & $17.9 \%$ \\
\hline \multicolumn{4}{|c|}{ Total direct plus indirect costs } \\
\hline First episodes $(422,950)$ & 126,885 & & \\
\hline I to $2(104,150)$ & 50,773 & & \\
\hline 3 to $6(468,650)$ & 712,817 & & \\
\hline \multirow[t]{2}{*}{7 or more $(124,970)$} & 307,051 & & \\
\hline & $1,197,526$ & & $100 \%$ \\
\hline
\end{tabular}

Note: Costs are expressed in 1996 US\$. 
Table 3: Average costs of genital herpes per patient in first and recurrent episodes

\begin{tabular}{|c|c|c|c|c|c|c|}
\hline & First episode & $\%$ & $\%$ of total & Recurrent episode & $\%$ & $\%$ of total \\
\hline \multicolumn{7}{|l|}{ Medical costs } \\
\hline Consultations & 62.6 & $35.8 \%$ & & 59.6 & $47.3 \%$ & \\
\hline Clinical examination & 39.8 & $22.7 \%$ & & 36.2 & $28.7 \%$ & \\
\hline Microbiologic tests & 17.6 & $10.1 \%$ & & 6.7 & $5.3 \%$ & \\
\hline Antibody testing & 12.8 & $7.3 \%$ & & 6.5 & $5.2 \%$ & \\
\hline Complete blood count & 4.6 & $2.6 \%$ & & 1.5 & $1.2 \%$ & \\
\hline Urine analysis & 4.6 & $2.6 \%$ & & 3.2 & $2.5 \%$ & \\
\hline Others & 33.0 & $18.9 \%$ & & 12.3 & $9.8 \%$ & \\
\hline Total & 175.0 & $100 \%$ & $58.3 \%$ & 126.0 & $100 \%$ & $38.8 \%$ \\
\hline \multicolumn{7}{|l|}{ Drug treatment } \\
\hline Antiviral treatment & 64.0 & $100 \%$ & & 131.0 & $100 \%$ & \\
\hline Total & 64.0 & $100 \%$ & $21.3 \%$ & 131.0 & $100 \%$ & $40.3 \%$ \\
\hline \multicolumn{7}{|l|}{ Hospital } \\
\hline Hospitalization & 1.0 & & $0.4 \%$ & 8.0 & & $2.4 \%$ \\
\hline Total direct costs & 240.0 & & $80.0 \%$ & 265.0 & & $81.5 \%$ \\
\hline \multicolumn{7}{|l|}{ Indirect costs } \\
\hline Production losses & 60.0 & & $20.0 \%$ & 60.0 & & $18.5 \%$ \\
\hline Total costs per patient & 300.0 & & $100 \%$ & 325.0 & & $100 \%$ \\
\hline
\end{tabular}

Note: Costs are expressed in 1996 US\$.

Table 4: Epidemiology of genital herpes in the claims database

\begin{tabular}{|c|c|c|c|c|c|c|c|c|c|c|}
\hline Age & Sex & Population & $\begin{array}{l}\text { Prevalent } \\
\text { GH }\end{array}$ & & $\begin{array}{l}\text { Incident } \\
\text { GH }\end{array}$ & & $\begin{array}{l}\text { Recur- } \\
\text { rent GH }\end{array}$ & & $\begin{array}{l}\text { Activ } \\
\text { GH }\end{array}$ & \\
\hline & & & $\mathbf{N}$ & $\begin{array}{l}\text { proportion/ } \\
\text { I,000 }\end{array}$ & $\mathbf{N}$ & rate $/ I, 000$ & $\mathbf{N}$ & rate/I,000 & $\mathbf{N}$ & rate $/ I, 000$ \\
\hline \multirow[t]{3}{*}{$0-20$} & Male & 81,966 & 12 & 0.15 & 3 & 0.04 & 0 & 0 & 4 & 0.05 \\
\hline & Female & 80,180 & 57 & 0.71 & 18 & 0.22 & 4 & 0.05 & 18 & 0.22 \\
\hline & Total & 162,146 & 69 & 0.43 & 21 & 0.14 & 4 & 0.02 & 22 & 0.14 \\
\hline \multirow[t]{3}{*}{$21-40$} & Male & 70,026 & 141 & 2.01 & 32 & 0.46 & 21 & 0.30 & 58 & 0.83 \\
\hline & Female & 84,067 & 455 & $5.4 I$ & 105 & 1.25 & 42 & 0.50 & 162 & 1.93 \\
\hline & Total & 154,093 & 596 & 3.87 & 137 & 0.89 & 63 & 0.41 & 220 & 1.43 \\
\hline
\end{tabular}


Table 4: Epidemiology of genital herpes in the claims database (Continued)

\begin{tabular}{|c|c|c|c|c|c|c|c|c|c|c|}
\hline \multirow[t]{3}{*}{$41-60$} & Male & 61,450 & 71 & 1.16 & 18 & 0.29 & 10 & 0.16 & 31 & 0.50 \\
\hline & Female & 69,947 & 159 & 2.27 & 43 & 0.61 & 25 & 0.36 & 62 & 0.89 \\
\hline & Total & 131,397 & 230 & 1.75 & 61 & 0.46 & 35 & 0.27 & 93 & 0.7 I \\
\hline \multirow[t]{3}{*}{$>60$} & Male & 9,045 & 3 & 0.33 & I & 0.11 & I & 0.11 & I & 0.11 \\
\hline & Female & 8,394 & 5 & 0.6 & 0 & 0 & I & 0.12 & 2 & 0.24 \\
\hline & Total & 17,439 & 8 & 0.46 & I & 0.06 & 2 & 0.11 & 3 & 0.17 \\
\hline \multirow[t]{3}{*}{ All } & Male & 222,487 & 227 & 1.02 & 53 & 0.24 & 32 & 0.14 & 94 & 0.42 \\
\hline & Female & 242,588 & 676 & 2.79 & 167 & 0.69 & 72 & 0.30 & 244 & 1.01 \\
\hline & Total & 465,075 & 903 & 1.94 & 220 & 0.47 & 104 & 0.22 & 338 & 0.73 \\
\hline
\end{tabular}

Table 5: Direct medical costs of genital herpes among claims database enrollees and extrapolated US estimates

\begin{tabular}{lcccc}
\hline Type of patient & Mean cost (\$) & SD (\$) & $\begin{array}{c}\text { Median cost } \\
\text { (\$) }\end{array}$ & $\begin{array}{c}\text { Mean total US costs } \\
\text { (in Mio \$) }\end{array}$ \\
\hline & & & & \\
Incident GH & & & 86 & 30.8 \\
Prevalent GH & 235 & 318 & 43 & 89.8 \\
Active GH & 166 & 536 & 191 & 21.8 \\
Recurrent GH & 355 & 707 & 359 & 101.6 \\
\hline
\end{tabular}

Note: Costs are expressed in 1996 US\$.

From an economic point of view, it must be borne in mind that GH and other sexually transmitted infectious diseases have negative externalities in the sense that consequences of the disease are not only limited to people who have the disease but also to other people that can be potentially infected. This stems from the fact that consequences of risky sexual behavior are borne by the subject itself and by others via the transmission of the disease. In addition, it must be underlined that GH constitutes a risk factor for the spread of other sexually transmitted diseases (e.g. human immunodeficency virus), which can be interpreted as a negative consequence of $\mathrm{GH}$.

The estimates of the total direct medical costs obtained with the two different approaches are discrepant and can be explained, at least in part, with the influence of compliance to treatment. It must be noted that the higher figure is obtained with data collected via questionnaire and is likely to represent the monetary value of the amount of treatment prescribed by physicians. Moreover, the reported duration of primary and recurrent GH episodes in this analysis was longer than that commonly cited in the literature $[5,8,17]$, which may have artificially increased the cost estimates. The lower figure, on the other hand, is an estimate based on claims and represents the mini- mum amount of medical care and treatment actually consumed by patients. This difference can be expressed as the difference from what is prescribed and what is actually consumed, i.e. compliance. Thus, the difference between the two global figures may be attributable to different utilization rates or different levels of compliance. A lower level of compliance means probably lower shortterm direct costs, but probably higher indirect and longterm medical costs. The hypothesis of different utilization rates is also consistent with the psychological aspects of $\mathrm{GH}$, which is perceived as a potential source of shame on patients [18] and is a plausible reason for lower levels of compliance to treatment.

In a recently published article by Tao et al [16], the national direct medical costs of $\mathrm{GH}$ were estimated at $\$ 166$ million annually for 1992-1994 (\$207 million in 1999 dollars), based on claims data from several sources. These numbers may be underestimates. Tao and colleagues [16] estimated that less than 30\% of acyclovir claims not associated with a specific diagnostic code were provided for the treatment of GH. As drugs account for over half of the costs attributed to $\mathrm{GH}$, underestimation of drug costs substantially decreases the estimated annual costs of $\mathrm{GH}$. 
Based on our estimates using the DPS claims database, GH seems to be a public health problem of important economic relevance. At least $\$ 283$ million can be estimated the direct health care costs attributable to $\mathrm{GH}$, corresponding to $0.1 \%$ of the US health care expenditure ( $\$ 1,007,300$ million). However, when computing for indirect costs and long-term complications (e.g. neonatal herpes, enhanced HIV transmission), the true costs may be greater than $\$ 1.0$ billion. In addition, this study has not been designed to give a monetary estimate of intangible costs of the disease. Psychological stress related to $\mathrm{GH}$ is well documented in the literature $[18,19,20]$, and should be considered as a relevant factor of the total burden of illness, though not easily quantifiable.

As with any research study, limitations must he placed on the ability to generalize the results beyond the sample and setting employed. First, treatment for GH in the USA can be met at neighborhood health clinics, which offer confidential, low-cost treatment. Since a stigma is attached to the diagnosis of $\mathrm{GH}$, patients may choose treatment at these clinics. The database had no information on these visits, since no claims were generated. Second, claims databases are collected for the purpose of payment to providers for the medical services rendered on behalf of enrolled members and rely on the coding of numerous medical events. Because of variations and incompleteness of coding, errors in identification and classification may occur. Coding is dependent on the diagnostic process, which is related to a clinician's training. Thus, the decision to diagnose GH by individual clinicians with different levels of expertise cannot be controlled within the boundaries of claims data. Third, the health plans included in this study allowed for geographical representation on a large regional basis. However, undetected patient, provider and practice differences may still exist. Caution should therefore be exercised in generalizing to other regions. Finally, cases selected may not necessarily be indicative of minority, low socioeconomic status, or indigent populations, since claims data can only provide data on those individuals who access the system. Therefore, the demographics of the database, in combination with the use of neighborhood clinics for GH treatment, make our calculated GH rates and costs lower bound estimates of the true GH prevalence and associated costs in the USA.

In conclusion, GH appears to be an important public health problem in the USA from the health economic point of view. The present study demonstrates the validity of using different approaches in analyzing the economic burden of a specific disease to the health care system.

\section{Competing interests}

TDS and KB have received funding from SmithKline Beecham Biologicals Ltd.

\section{References}

I. Whitley RJ, Kimberlin DW, Roizman B: Herpes simplex viruses Clin Infect Dis 1998, 26(3):54I-553

2. Corey L, Handsfield HH: Genital herpes and public health: addressing a global problem JAMA 2000, 283(6):79I-794

3. Fleming DT, McQuillan GM, Johnson RE, Nahmias AJ, Aral SO, Lee FK, et al: Herpes simplex virus type 2 in the United States, I 976 to I994 N Engl J Med I997, 337( I6): I I05- I I I I

4. Brugha R, Keersmaekers K, Renton A, Meheus A: Genital herpes infection: a review Int J Epidemiol 1997, 26(4):698-709

5. Benedetti J, Corey L, Ashley R: Recurrence rates in genital herpes after symptomatic first-episode infection Ann Intern Med I994, I 2 I ( I I ):847-854

6. Behrens C, Henke K: Cost of illness studies: no aid to decision making Health Policy 1988, I0(2):|37-|4|

7. Rice DP: Cost-of-illness studies: fact or fiction? Lancet 1994, 344(8936): $1519-1520$

8. Stanberry LR, Cunningham AL, Mindel A, Scott LL, Spruance SL, Aoki FY, et al: Prospects for control of herpes simplex virus disease through immunization Clin Infect Dis 2000, 30(3):549-566

9. Buxton MJ, Drummond MF, Van Hout BA, Prince RL, Sheldon TA, Szucs T, et al: Health Econ 1997, 6(3):217-227

10. Koopmanschap MA, Rutten FF: Indirect costs in economic studies: confronting the confusion Pharmacoeconomics 1993, 4(6):446-454

II. Drummond MF, Richardson WS, O'Brien BJ, Levine M, Heyland D: Users' guides to the medical literature. XIII. How to use an article on economic analysis of clinical practice. A. Are the results of the study valid? JAMA 1997, 277(19): I552-I557

12. Bureau of Labor Statistics. Selective Data: U.S. Department of Labor; 1999 [http://stats.bls.gov.80/sahome.html]

13. Randolph AG, Washington AE, Prober CG: Cesarean delivery for women presenting with genital herpes lesions. Efficacy, risks, and costs JAMA 1993, 270(I):77-82

14. Randolph AG, Hartshorn RM, Washington AE: Acyclovir prophylaxis in late pregnancy to prevent neonatal herpes: a cost-effectiveness analysis Obstet Gynecol 1996, 88(4 Pt I):603-610

15. Scott LL, Alexander J: Cost-effectiveness of acyclovir suppression to prevent recurrent genital herpes in term pregnancy Am J Perinatol 1998, I 5(I):57-62

16. Tao G, Kassler WJ, Rein DB: Medical care expenditures for genital herpes in the United States Sex Transm Dis 2000, 27( I):32-38

17. Wald A, Zeh J, Selke S, Warren T, Ryncarz AJ, Ashley R, et al: Reactivation of genital herpes simplex virus type $\mathbf{2}$ infection in asymptomatic seropositive persons $N$ Engl J Med 2000 , 342(12):844-850

18. Mindel A: Psychological and psychosexual implications of herpes simplex virus infections Scand J Infect Dis Suppl 1996, 100:2732

19. Luby ED, Klinge V: Genital herpes. A pervasive psychosocial disorder Arch Dermatol I985, I 2 I (4):494-7

20. Goldmeier D, Johnson A, Byrne M, Barton S: Psychosocial implications of recurrent genital herpes simplex virus infection Genitourin Med I 988, 64(5):327-330

\section{Pre-publication history}

The pre-publication history for this paper can be accessed here:

http://www.biomedcentral.com/content/backmatter/ 1471-2334-1-5-b1.pdf 